\title{
Patient-Derived Tumor Organoids: A Promising Tool for Personalized Cancer Therapy
}

\section{Divya Sasidharan Padmaja and Pratheeshkumar Poyil}

Human Cancer Genomic Research, King Faisal Specialist Hospital and Research Center, Saudi Arabia

"Corresponding author: Pratheesh kumar Poyil, Human Cancer Genomic Research, King Faisal Specialist Hospital and Research Center, Riyadh, Saudi Arabia, Tel: 8594948328; E-mail: ppratheeshkumar@gmail.com

Received date: May 16, 2018; Accepted date: May 28, 2018; Published date: June 04, 2018

Copyright: (C2018 Padmaja DS, et al. This is an open-access article distributed under the terms of the Creative Commons Attribution License, which permits unrestricted use, distribution, and reproduction in any medium, provided the original author and source are credited.

Citation: Padmaja DS, Poyil P(2018) Patient-Derived Tumor Organoids: A Promising Tool for Personalized Cancer Therapy. J Carcinog Mutagen 9: 319. doi: $10.4172 / 2157-2518.1000 \mathrm{e} 124$

\section{Editorial}

Patient Derived Organoids (PDOs) are described as miniature, three dimensional (3D) cell cultures prepared from patient's cancer cell to compare therapeutic responses in the laboratory and clinical settings. Personalized cancer medicine is a novel therapeutic strategy to identify a right treatment for the right patient in accordance with tumor's genetic signature. Tumor Organoid models have innumerable benefits over pre-existing models which makes them a tremendous candidate in personalized cancer therapy as they mimic the physiology of the original tumor. Recently several studies have shown the value of these "tumor in-dish" approaches in personalized cancer medicine for preclinical drug screening and prediction of patient treatment outcome. The objective of this "Journal of Carcinogenesis and Mutagenesis" research topic is to advance our current understanding about PDOs as an attractive in vitro model system for studying tumor evolution and response to drugs and treatments.

The study team composed of researchers from Rutgers Cancer Institute and Harvard Medical School, Boston, USA describe an interdisciplinary approach for using primary prostate cancer and tumor initiating cells, 3D-tissue engineering and humanized mice to generate PDOs for exploring personalized therapeutic strategy in vitro $[1,2]$ emphasize in their review "Personalized Cancer Medicine: An Organoid Approach" that there is an urgent need for research in personalized cancer therapy to predict drug response from the genomic and transcriptomic features of cancer in the preclinical settings. The research team led by Hans Clevers and Marc Van de Watering from Hubrecht Institute, Netherlands has elegantly established PDOs from twenty colorectal cancer (CRC) patients [3]. These PDOs showed similar pattern of genetic changes with parental CRC tissue and are amenable to high throughput drug screening permitting detection of gene-drug associations.

Writing in Science, Vlachogiannis and colleagues [4] have successfully made living organoid biobank from metastatic gastrointestinal (GI) cancer patients who have already enrolled in clinical trial phase I or II. Encouragingly the authors have found that these PDOs had same mutation pattern to those of patient tumor. Moreover, the researchers studied the possibility of using PDOs as a drug screening model by investigating twenty-one assessments of patient clinical drug responses to ex-vivo organoid responses. Interestingly, the researchers reported response to targeted drugs between PDOs and patients showed significantly high sensitivity (100\%), specificity (93\%), positive predictive value ( $88 \%)$ and negative predictive value $(100 \%)$.

In another study, Lee et al. [5], established a biobank of PDOs from human bladder cancer patient's biopsies ranging from low grade nonmuscle invasive disease to high grade muscle invasive cancers and observed that PDOs could successfully recapitulate the broad histopathological and molecular spectrum of both type of tumors with their corresponding parental tumors. A detailed analysis made on their mutational profile displayed considerable changes during culture and xenografting that are consistent with the clonal evolution.

In summary, PDOs model represents a rapid and cost-effective potential tool for personalized cancer therapy for preclinical drug screening and predict individual treatment response.

\section{References}

1. Bartucci M, Ferrari AC, Kim IY, Ploss A, Yarmush M, et al. (2016) Personalized Medicine Approaches in Prostate Cancer Employing Patient Derived 3D Organoids and Humanized Mice. Front Cell Dev Biol 4: 74.

2. Aboulkheyr EH, Montazeri L, Aref AR, Vosough M, Baharvand H (2018) Personalized Cancer Medicine: An Organoid Approach. Trends Biotechnol 36: 358-371.

3. van de Wetering M, Francies HE, Francis JM, Bounova G, Iorio F, et al. (2015) Prospective derivation of a living organoid biobank of colorectal cancer patients. Cell 161: 933-945.

4. Vlachogiannis G, Hedayat S, Vatsiou A, Jamin Y, Fernández Mateos J, et al. (2018) Patient-derived organoids model treatment response of metastatic gastrointestinal cancers. Science 359: 920-926.

5. Lee SH, Hu W, Matulay JT, Silva MV, Owczarek TB, et al. (2018) Tumor Evolution and Drug Response in Patient-Derived Organoid Models of Bladder Cancer. Cell 173: 515-528. 